\title{
Epileptic Seizure Provoked by Bone Metastasis of Chronic Lymphoid Leukemia and Merkel Cell Carcinoma - Case Report
}

\section{András Folyovich}

Szent János Kórház

Angéla Majoros

Szent János Kórház

Tamás Jarecsny

Szent János Korhaz

\section{Gitta Pánczél}

Orszagos Onkologiai Intezet

\section{Zsuzsanna Pápai}

Orszagos Onkologiai Intezet

\section{Gábor Rudas}

Semmelweis Egyetem

\section{Lajos Kozák}

Semmelweis Egyetem

\section{Gábor Barna}

Semmelweis Egyetem

Katalin A. Béres-Molnár

Szent János Korhaz

Károly Vadasdi

Orszagos Onkologiai Intezet

Gabriella Liszkay

Orszagos Onkologiai Intezet

Gergely Toldi ( $\nabla$ toldigergely@yahoo.com )

Semmelweis University https://orcid.org/0000-0003-0178-1243

\section{Case report}

Keywords: Bone metastasis, chronic lymphoid leukemia, epileptic seizure, Merkel cell carcinoma

Posted Date: February 20th, 2019

DOI: https://doi.org/10.21203/rs.2.358/v1 
License: (c) (i) This work is licensed under a Creative Commons Attribution 4.0 International License. Read Full License 


\section{Abstract}

Background Merkel cell carcinoma (MCC) is a rare primary neuroendocrine cutaneous tumor, rarely metastatizing to the brain. Chronic lymphoid leukemia (CLL) is a disease predisposing to MCC. According to previous reports, headache and focal neurological deficits suggest disease progression to the brain. We present a patient with MCC whose seizure was not elicited by a cerebral metastasis, but by bone metastases compressing the brain. Case presentation The 62-year-old female patient had a history of CLL. A lesion with the appearance of an atheroma was removed from the right upper arm. Histology confirmed the diagnosis of MCC. She was admitted to the neurology department with her first GM seizure. The cranial MRI/MRA showed bone metastases in the right parietal and left frontal areas, compressing the brain. Flow cytometry of CSF did not reveal metastasis of MCC or CLL. No surgery was performed, chemotherapy was continued. Conclusions The case history of the patient was unique even among the rare cases of MCC with neurological involvement. The seizure was not elicited by a cerebral metastasis, but by bone metastases compressing the brain. In addition to patient history, clinical presentation and radiological findings enabled a suspected diagnosis preceding confirmation by laboratory methods.

\section{Background}

Merkel cell carcinoma (MCC) is a rare and extremely aggressive neuroendocrine malignancy. Both clinically and histologically, it is difficult to differentiate it from neuroendocrine carcinomas, skin metastasis of small cell lung cancer, small cell malignant melanoma, malignant lymphoma, atypical keratoacanthoma and leiomyosarcoma. In addition to patient history, clinical and radiological findings, as well as immunohistochemistry assist in making a definite diagnosis. Due to the above reasons, it is a significant challenge for the neurologist to diagnose this very rare and malignant disease in a timely manner which needs rapid treatment to have a chance of recovery.

\section{Case Presentation}

The 62-year-old female patient's chronic lymphoid leukemia (CLL) was diagnosed 10 years earlier and was non-progressive under treatment. A lesion with the appearance of an atheroma was removed from the right upper arm 2 years before her admission to the Department of Neurology. The lesion recurred and was reoperated. Histology with immunohistochemistry confirmed the diagnosis MCC. Sentinel lymph node biopsy did not show infiltration from MCC, but progression of CLL. The patient later had right axillary block dissection which showed metastases from both CLL and MCC. Combined chemotherapy based on National Comprehensive Cancer Network (NCCN) guidelines was discontinued because of bone marrow depression. Postoperative radiotherapy was performed, and etoposide and cisplatin chemotherapy of CLL was continued. The patient's condition deteriorated. A right axillary soft tissue conglomerate mass infiltrated the right lateral thoracic wall and pathologic upper mediastinal and parasternal lymph nodes appeared. She was admitted to our Department of Neurology due to her first 
epileptic seizure (GM). The last chemotherapy session (vincristin) was administered 3 days earlier. The patient complained about headache during the week before admission.

General medical state: BP: 115/75 mmHg, HR: 75/min. No bruit over carotid or subclavian arteries. An oblique surgical scar with primary healing on the right upper arm. Liver edge at the costal margin. No other abnormality was found during physical examination.

Neurological status: Normally shaped skull. No meningeal signs. Intact cranial nerves. Symmetric, normal deep tendon reflexes. No pathologic reflexes. Intact motor and sensory system, and coordination. No aphasia or lobe syndrome. Normal vigilance.

Mental state: no abnormality was found.

Laboratory findings: WBC: 11.48 10*9/L; Neutrophils (\%): 5.9, ratio of stab cells: 1\%; Lymphocytes (\%): 86.4\%; Monocytes (\%): 1.0\%; Eosinophiles (\%): 0.1\%; Basophiles (\%): 0.4\%; RBC: 3.90 10*12/L; Hgb: 112 g/L; Hct: 0.335 L/L; Platelets: 85 10*9/L; Blood smear: Gumprecth's shadows.

Normal serum glucose, creatinine and electrolyte levels. Gamma-GT: 24 U/L; Alkaline phosphatase: 162 U/L; LDH: 1768 U/L; Amylase: 75 U/L; CRP: 1.59 mg/L.

Cranial CT: showed a $37 \times 9 \mathrm{~mm}$ hypodensity in the right parietal region, at the posterior horn of the lateral ventricle, with marked cerebral and cerebellar satrophy.

Cranial MR (axial FLAIR, T2, sagittal 3D T1 (with and without contrast application): a solid epidural $2 \times 2.8 \times 5 \mathrm{~cm}$ mass, originating from the bone, in the right parietal region, showing homogeneously low signal intensity on T1-weighted images (Figure 1/a) and homogeneously high signal intensity on T2 and FLAIR images (Figure 1/b), with an intense contrast enhancement (mainly) in the peripheral zone, and smaller necrotic areas in the central zone. Moderate oedema around the mass is present, with higher apparent diffusion coefficient (ADC) value on the ADC images. A $6.2 \times 3.2 \times 1.3 \mathrm{~cm}$ mass, infiltrating the bone, with similar structure and contrast enhancement is seen in mainly the left frontal area (Figure 1/cd). The mass shows higher ADC values in diffusion images. Lacunar encephalopathy, cerebral and cerebellar atrophy were also noted.

Lumbar cerebrospinal fluid (CSF): Clear and transparent; Cell count: 5 WBC/microliter, Total protein: 368 $\mathrm{mg} / \mathrm{L}$; CSF glucose: $3.8 \mathrm{mmol} / \mathrm{L}$; Serum glucose (simultaneous): $7.5 \mathrm{mmol} / \mathrm{L}$

CSF cytology: few monocytoid cells and leukocytes. No tumor cells are detected in the sample.

CSF immunology: intact blood-liquor barrier. Intrathecal IgG synthesis is not detected, but oligoclonal gammopathy (OGP) is found.

Flow cytometry of CSF did not reveal metastasis of MCC. CLL was only present in peripheral blood, where $50 \%$ of the cells showed a corresponding flow cytometry phenotype. 
Based on the clinical picture, the patient was started on carbamazepine. She did not have further epileptic seizures, and did not develop any neurological deficit. During an oncology follow-up visit, the patient announced that she and her husband had decided to continue her treatment abroad. We have no knowledge of her since then.

The diagnosis at the time of discharge was: Chronic lymphoid leukemia, Merkel cell carcinoma, metastatic bone tumors of bilateral frontal bones and right parietal bone, symptomatic epilepsy, lacunar encephalopathy, cerebral and cerebellar atrophy.

\section{Discussion And Conclusions}

MCC is a rare and extremely aggressive neuroendocrine malignancy, presenting as a solitary, painless, thick, livid and erythematous nodule, mostly on skin areas exposed to sunlight. First, it gives metastases to regional lymph nodes and then to distant organs. MCC is often associated with malignant hematologic diseases, such as CLL [1]. Recently, a polyomavirus was identified, likely to be involved in the pathogenesis of MCC [2]. Genetic instability and impaired immune defence due to the primary malignancy contribute to the development of other solid tumors, which may explain the coexistence of MCC with CLL. In addition to conventional radiochemotherapy, novel therapeutic options include treatment with somatostatin analogues in neuroendocrine tumors expressing somatostatin receptors, monoclonal antibody therapy in tumors expressing CD56 antigens, and anti-PD-L1 antibody therapy.

The case history of our patient is unique even among rare cases of MCC giving metastases to the brain $[3,4]$ due to the following factors:

1. She had a history of proven CLL.

2. The seizure was not elicited by a cerebral metastasis, but by bone metastases compressing the brain.

In addition to patient history, clinical presentation and radiological findings enabled a suspected diagnosis preceding confirmation by laboratory methods.

\section{Abbreviations}

ADC apparent diffusion coefficient

CLL chronic lymphoid leukemia

CSF cerebrospinal fluid

MCC Merkel cell carcinoma

\section{Declarations}


Ethics approval and consent to participate

N/A

Consent for publication

Consent requested from patient's relatives as patient deceased

Availability of data and material

$\mathrm{N} / \mathrm{A}$

Competing interests

The authors declare that they have no competing interests.

Funding

N/A

Authors' contributions

$\mathrm{AF}$ and $\mathrm{GT}$ wrote manuscript. AF, AM, TJ, KAB-M and $\mathrm{KV}$ were responsible for neurological management of the patient and critically read manuscript. GP, ZP and GL were responsible for oncological management of the patient and critically read manuscript. GR and LK performed and interpreted imaging studies and critically read manuscript. GB performed and interpreted flow cytometry studies and critically read manuscript.

Acknowledgements

N/A

CAREs checklist 


\section{References}

1. Tadmor T, Aviv A, Polliack A. Merkel cell carcinoma, chronic lymphocytic leukemia and other lymphoproliferative disorders: an old bond with possible new viral ties. Ann Oncol. 2011;22:250-6.

2. Koljonen V, Kukki H, Pukkala E, Sankila R, Böhling T, Tukiainen E, Sihto H, Joensuu H. Chronic lymphocytic leukaemia patients have a high risk of Merkel-cell polyomavirus DNA-positive Merkelcell carcinoma. Br J Cancer. 2009;101:1444-7.

3. Abul-Kasim K, Söderström K, Hallsten L. Extensive central nervous system involvement in Merkel cell carcinoma: a case report and review of the literature. J Med Case Rep. 2011;5:35.

4. Eggers SD, Salomao DR, Dinapoli RP, Vernino S. Paraneoplastic and metastatic neurologic complications of Merkel cell carcinoma. Mayo Clin Proc. 2001;76:327-30.

\section{Figures}
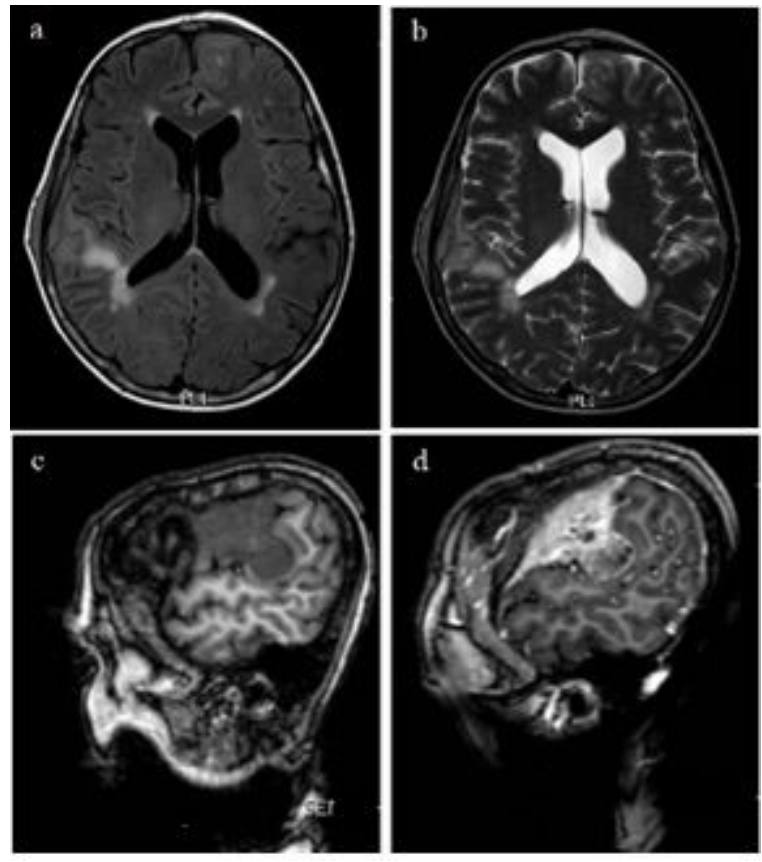

Figure 1

Cranial MR on admission to the Department of Neurology. Axial FLAIR, T2, sagittal 3D T1 (with and without contrast application) - Philips Achieva 3 Tesla.

\section{Supplementary Files}

This is a list of supplementary files associated with this preprint. Click to download. 
- supplement1.pdf

Page 8/8 